International Journal of Pure and Applied Mathematics

Volume 109 No. $4 \quad 2016,837-846$

ISSN: 1311-8080 (printed version); ISSN: 1314-3395 (on-line version)

url: http://www.ijpam.eu

doi: 10.12732/ijpam.v109i4.7

ijpam.eu

\title{
BIPOLAR VALUED FUZZY IDEALS OF BF-ALGEBRAS
}

\author{
Shanmugavelu Sabarinathan ${ }^{1 \S}$, David C. Kumar ${ }^{2}$, Prakasam Muralikrishna ${ }^{3}$ \\ ${ }^{1}$ Department of Mathematics \\ Latha Mathavan Engineering College \\ Madurai, Tamilnadu, INDIA \\ ${ }^{2}$ Department of Mathematics \\ Vickram College of Engineering \\ Enathi, 630561, Tamilnadu, INDIA \\ ${ }^{3} \mathrm{PG} \&$ Research Department of Mathematics \\ Muthurangam Government Arts College \\ Vellore, 632002, Tamilnadu, INDIA
}

AMS Subject Classification: 06F35, 03G25, 08A72, 03E72

Key Words: $B F$-algebra, subalgebra, ideal, bipolar valued fuzzy subalgebra, bipolar valued fuzzy ideal

\section{Introduction}

In the traditional fuzzy sets [7], the membership degrees of elements range over the interval $[0,1]$. Lee[3] has introduced an extension of fuzzy sets named bipolar valued-valued fuzzy sets in 2000 and in [4], he compares it with other fuzzy settings . Bipolar valued fuzzy sets are an extension of fuzzy sets whose membership degree range is enlarged from the interval $[0,1]$ to $[-1,1]$. Bipolar valued fuzzy sets have membership degrees that represent the degree of satisfication to the property corresponding to a fuzzy set and its counter-property in a bipolar valued fuzzy set, the membership degree 0 means that the elements are irrele-

Received: $\quad$ August 1, 2016

Revised: $\quad$ August 19, 2016

(c) 2016 Academic Publications, Ltd.

Published: October 6, 2016

url: www.acadpubl.eu

$\S_{\text {Correspondence author }}$ 
vant to the corresponding property. Further the membership degrees on $(0,1]$ indicate that the elements somewhat satisfy the property, and the membership degrees on $[-1,0)$ indicate that elements somewhat satisfy the implicit counter property.

In the definition of bipolar valued fuzzy sets, there are two kinds of representations, such as canonical representation and reduced representation. Here, the canonical representation of a bipolar valued fuzzy sets is used. Let $X$ be the universe of discourse.

In [5] and [6], the authors dealt the concepts of Homomorphic image of Intuitionistic L-Fuzzy ideals and N-ideals of BF-algebras respectively. In 2011, Farhat Nisar[2], discussed the Bipolar-valued Fuzzy K-subalgebras. Motivated by this, in this paper, the notion of Bipolar Valued Fuzzy Set (BVFS) is applied in BF-algebras[1]. The concept of bipolar valued fuzzy subalgebras/ideals of a $\mathrm{BF}$-algebra is introduced and several properties are investigated. The relations between a bipolar valued fuzzy subalgebra and a bipolar valued fuzzy ideal are given. A condition is provided for a bipolar valued fuzzy subalgebra to be a bipolar valued fuzzy ideal. The characterizations of a bipolar valued fuzzy ideal is also given. The concept of eqivalence relations on the family of all bipolar valued fuzzy ideals of BF-algebra is considered and some related properties are analysed.

The paper has been organised as follows: Section 2 provides the preliminaries. Section 3 deals with the notion of Bipolar valued Fuzzy Subalgebra. Section 4 discusses the Bipolar valued Fuzzy ideal of BF-algebras and the conclusion is presented in Section 5.

\section{Preliminaries}

In this section some basic definitions and results that are needed in the sequel are recalled. The following notations are also used in the rest of the paper: $\min (a, b)=a \wedge b$ and $\max (a, b)=a \vee b$.

\subsection{Basic Results on BF-Algebras}

Definition 1. [1] A BF-algebra is a non-empty set $X$ with a constant 0 and a single binary operation $*$ which satisfies the following axioms:

1. $x * x=0$.

2. $x * 0=x$. 
3. $0 *(x * y)=y * x$, for all $x, y \in X$.

Example 2. Let $X=\{0,1,2,3,4\}$ be a set with the following table.

\begin{tabular}{|c|c|c|c|c|c|}
\hline$*$ & 0 & 1 & 2 & 3 & 4 \\
\hline 0 & 0 & 4 & 3 & 2 & 1 \\
\hline 1 & 1 & 0 & 4 & 3 & 2 \\
\hline 2 & 2 & 1 & 0 & 4 & 3 \\
\hline 3 & 3 & 2 & 1 & 0 & 4 \\
\hline 4 & 4 & 3 & 2 & 1 & 0 \\
\hline
\end{tabular}

Then $(X, *, 0)$ is BF-algebra.

Definition 3. [1] A BG-algebra is a non-empty set $X$ with a constant 0 and a single binary operation $*$ which satisfies the following axioms:

1. $x * x=0$.

2. $x * 0=x$.

3. $(x * y) *(0 * y)=x$ for all $x, y \in X$.

A binary relation $\leq$ on a BF-algebra $X$ can be defined as $x \leq y$, if and only if $x * y=0$. A subset $S$ of a BF-algebra $X$ is called a subalgebra of $X$ if $x * y \in S$ for all $x, y \in S$. An ideal of a BF-algebra $X$ is a subset $I$ of $X$ containing 0 such that if $x * y \in I$ and $y \in I$, then $x \in I$. Note that every ideal of a BF-algebra $X$ has the following property: $x \leq y$ and $y \in I$ imply $x \in I$.

A fuzzy set $\mu$ in a BF-algebra $X$ is said to be a fuzzy subalgebra of $X$, if it satisfies:

$$
\mu(x * y) \geq \mu(x) \wedge \mu(y) \text { for all } x, y \in X
$$

A fuzzy set $\mu$ in a BF-algebra $X$ is said to be a fuzzy ideal of $X$, if it satisfies:

$$
\begin{aligned}
& \mu(0) \geq \mu(x) \text { for all } x \in X \\
& \mu(x) \geq \mu(x * y) \wedge \mu(y) \text { for all } x, y \in X
\end{aligned}
$$

Note that every fuzzy ideal $\mu$ of a BF-algebra $X$ is order reversing, i.e., if $x \leq y$, then $\mu(x) \geq \mu(y)$.

An ideal $I$ of $X$ is called closed, if $0 * x \in I \forall x \in I$. 


\subsection{Basic Results on Bipolar Valued Fuzzy Set}

Fuzzy sets are useful mathematical structure to represent a collection of objects and whose boundary is vague. There are several kinds of fuzzy set extensions in the fuzzy set theory, for example, intuitionistic fuzzy sets, interval-valued fuzzy sets, vague sets,etc.

Definition 4. Let $X$ be a non empty set. A Bipolar Valued Fuzzy Set (BVFS) $B$ in $X$ is an object with the form

$$
B=\left\{\left(x ; \mu^{+}(x), \nu^{-}(x)\right) \mid x \in X\right\}
$$

where $\mu^{+}: X \rightarrow[0,1]$ and $\nu^{-}: X \rightarrow[-1,0]$ are mappings.

The positive membership degree $\mu^{+}(x)$ denotes the satisfication degree of an element $x$ to the property corresponding to a bipolar valued fuzzy set $B=$ $\left\{\left(x ; \mu^{+}(x), \nu^{-}(x)\right) \mid x \in X\right\}$ and the negative membership degree $\nu^{-}(x)$ denotes the satisfication degree of an element $x$ to some implicit counter-property corresponding to a bipolar valued-valued fuzzy set $B=\left\{\left(x: \mu^{+}(x), \nu^{-}(x)\right) \mid x \in X\right\}$. If $\mu^{+}(x) \neq 0$ and $\nu^{-}(x)=0$, it is the situation that $x$ is regarded as having only positive satisfication for $B=\left\{\left(x ; \mu^{+}(x), \nu^{-}(x)\right) \mid x \in X\right\}$ If $\mu^{+}(x)=0$ and $\nu^{-}(x) \neq 0$, it is the situation that $x$ does not satisfy the property of $B=\left\{\left(x ; \mu^{+}(x), \nu^{-}(x)\right) \mid x \in X\right\}$ but somewhat satisfies the counter property of $B=\left\{\left(x ; \mu^{+}(x), \nu^{-}(x)\right) \mid x \in X\right\}$. It is possible for an element $x$ to be such that $\mu^{+}(x) \neq 0$ and $\nu^{-}(x) \neq 0$, when the membership function of the property overlaps that of its counter property over some portion of $X$. For the sake of simplicity, the symbol $B=\left(X ; \mu^{+}, \nu^{-}\right)$shall be used for the bipolarvalued fuzzy set $B=\left\{\left(x ; \mu^{+}(x), \nu^{-}(x)\right) \mid x \in X\right\}$.

\section{Bipolar Valued Fuzzy Subalgebra}

In this section, the notion of Bipolar Valued Fuzzy Subalgebra of a BF-algebra is introduced and some elegant results are also discussed. In the forth coming sections, $X$ is denoted as BF-algebra unless otherwise specified.

Definition 5. A BVFS $B=\left(X ; \mu^{+}, \nu^{-}\right)$in $X$ is called a bipolar valued fuzzy subalgebra of $X$, which satisfies:

1. $\mu^{+}(x * y) \geq \mu^{+}(x) \wedge \mu^{+}(y)$

2. $\nu^{-}(x * y) \leq \nu^{-}(x) \vee \nu^{-}(y) \forall x, y \in X$ 
Example 6. A BF-algebra in example 2 is considered. Let $B=\left(X ; \mu^{+}, \nu^{-}\right)$be a BVFS in $X$ defined by

$$
\mu^{+}(x)=\left\{\begin{array}{l}
0.6 ; x \neq 2 \\
0.1 ; x=2
\end{array} \text { and } \nu^{-}(x)=\left\{\begin{array}{l}
-0.8 ; x \neq 2 \\
-0.2 ; x=2
\end{array}\right.\right.
$$

Then, $B=\left(X ; \mu^{+}, \nu^{-}\right)$is a bipolar valued fuzzy subalgebra of $X$.

Theorem 7. If $B=\left(X ; \mu^{+}, \nu^{-}\right)$is a bipolar valued fuzzy subalgebra of $X$, then $\mu^{+}(0) \geq \mu^{+}(x)$ and $\nu^{-}(0) \leq \nu^{-}(x)$ for all $x \in X$.

Proof. Let $x \in X$. Then,

$\mu^{+}(0)=\mu^{+}(x * x) \geq \mu^{+}(x) \wedge \mu^{+}(x)=\mu^{+}(x)$.

and

$\nu^{-}(0)=\nu^{-}(x * x) \leq \nu^{-}(x) \vee \nu^{-}(x)=\nu^{-}(x)$.

This completes the proof.

Definition 8. For a BVFS $B=\left(X ; \mu^{+}, \nu^{-}\right)$and $(s, t) \in[-1,0] \times[0,1]$, it is defined that $B_{t}^{+}=\left\{x \in X \mid \mu^{+}(x) \geq t\right\}$ and $B_{s}^{-}=\left\{x \in X \mid \nu^{-}(x) \leq s\right\}$ which are called the positive $t$-cut of $B=\left(X ; \mu^{+}, \nu^{-}\right)$and the negative $s-$ cut of $B=\left(X ; \mu^{+}, \nu^{-}\right)$, respectively.

For every $k \in[0,1]$, the set $B_{k}=B_{k}^{+} \cap B_{-k}^{-}$is called the $k$-cut of $B=$ $\left(X ; \mu^{+}, \nu^{-}\right)$.

Theorem 9. Let $B=\left(X, \mu^{+}, \nu^{-}\right)$be a bipolar valued fuzzy subalgebra of $X$. Then, the following assertions are valid.

1. For all $t \in[0,1]\left(B_{t}^{+} \neq \phi \Rightarrow B_{t}^{+}\right.$is a subalgebra of $\left.X\right)$.

2. For all $s \in[0,1]\left(\left(B_{s}^{-} \neq \phi \Rightarrow B_{s}^{-}\right.\right.$is a subalgebra of $X$. $)$

Proof. 1. Let $t \in[0,1]$ be such that $B_{t}^{+} \neq \phi$. If $x, y \in B_{t}^{+}$, then $\mu^{+}(x) \geq t$ and $\mu^{+}(y) \geq t$. It follows that

$$
\mu^{+}(x * y) \geq \mu^{+}(x) \wedge \mu^{+}(y) \geq t
$$

so that, $x * y \in B_{t}^{+}$. Therefore $B_{t}^{+}$is a subalgebra of $X$.

2. Now, let $s \in[-1,0]$ be such that $B_{s}^{-} \neq \phi$. If $x, y \in B_{s}^{-}$, then

$$
\nu^{-}(x * y) \leq \nu^{-}(x) \vee \nu^{-}(y) \leq s
$$

and so $x * y \in B_{s}^{-}$. Hence, $B_{s}^{-}$is a subalgebra of $X$. 
Corollary 10. If $B=\left(X ; \mu^{+}, \nu^{-}\right)$is a bipolar valued fuzzy subalgebra of $X$, then the sets $B_{\mu^{+}(0)}^{+}$and $B_{\nu^{-}(0)}^{-}$are subalgebras of $X$.

Proof. Straightforward.

\section{Bipolar Valued Fuzzy Ideal of BF-Algebras}

This section deals with the ideas of Bipolar Valued Fuzzy Ideal of BF-algebras.

Definition 11. A BVFS $B=\left(X ; \mu^{+}, \nu^{-}\right)$in $X$ is called a bipolar valued fuzzy ideal (BVF-ideal) of $X$, if it satisfies:

1. $\mu^{+}(0) \geq \mu^{+}(x)$ and $\nu^{-}(0) \leq \nu^{-}(x)$.

2. $\mu^{+}(x) \geq \mu^{+}(x * y) \wedge \mu^{+}(y)$

3. $\nu^{-}(x) \leq \nu^{-}(x * y) \vee \nu^{-}(y)$ for all $x, y \in X$.

Example 12. The BF-algebra $X=\{0,1,2,3,4\}$ is considered as in Example $2 . B=\left(X ; \mu^{+}, \nu^{-}\right)$is the BVFS of $X$ defined by

$$
\mu^{+}(x)=\left\{\begin{array}{ccc}
1 & ; & x=0,2 \\
0.4 & ; & x=1,3,4
\end{array} \quad \text { and } \quad \nu^{-}(x)=\left\{\begin{array}{cc}
-1 & ; \quad x=0,2 \\
-0.3 ; & x=1,3,4
\end{array}\right.\right.
$$

Then, $B=\left(X ; \mu^{+}, \nu^{-}\right)$is a BVF-ideal of $X$.

Definition 13. A BVFS $B$ in a BF-algebra $X$ is said to be a Bipolar valued fuzzy Closed-BF- ideal (BVFC-BF-ideal) of $X$, if

1. $\mu^{+}(x) \geq \mu^{+}(x * y) \wedge \mu^{+}(y)$

2. $\nu^{-}(x) \leq \nu^{-}(x * y) \vee \nu^{-}(y)$

3. $\mu^{+}(0 * x) \geq \mu^{+}(x)$

4. $\nu^{-}(0 * x) \leq \nu^{-}(x) \quad \forall x, y \in X$.

Example 14. The BF-algebra $X=\{0,1,2,3\}$ with the Cayley table given below is considered.

\begin{tabular}{|l|l|l|l|l|}
\hline$*$ & 0 & 1 & 2 & 3 \\
\hline 0 & 0 & 1 & 2 & 3 \\
\hline 1 & 1 & 0 & 3 & 2 \\
\hline 2 & 2 & 3 & 0 & 1 \\
\hline 3 & 3 & 2 & 1 & 0 \\
\hline
\end{tabular}


The BVFS of $X, B=\left(X ; \mu^{+}, \nu^{-}\right)$defined by

$$
\mu^{+}(x)=\left\{\begin{array}{cc}
0.8 & x=0 \\
0.5 & x=1 \\
0.3 & x=2,3
\end{array} \quad \text { and } \quad \nu^{-}(x)=\left\{\begin{array}{cc}
-0.4 & x=0 \\
-0.3 & x=1 \\
-0.1 & x=2,3
\end{array}\right.\right.
$$

is a BVFC-BF-ideal of $X$.

Proposition 15. Every BVFC-BF-ideal is a BVF-ideal.

Proof. It is clear.

In general, the converse of the above Proposition is not true as seen from the following:

Example 16. Consider the BF-algebra $X=\{0,1,2,3\}$ with the Cayley table given below.

\begin{tabular}{|c|c|c|c|c|}
\hline$*$ & 0 & 1 & 2 & 3 \\
\hline 0 & 0 & 3 & 0 & 1 \\
\hline 1 & 1 & 0 & 1 & 3 \\
\hline 2 & 2 & 3 & 0 & 1 \\
\hline 3 & 3 & 1 & 3 & 0 \\
\hline
\end{tabular}

Then, the BVFS of $X, B=\left(X ; \mu^{+}, \nu^{-}\right)$defined by

$$
\mu^{+}(x)=\left\{\begin{array}{cc}
0.8 & x=0 \\
0.5 & x=1 \\
0.3 & x=2,3
\end{array} \text { and } \quad \nu^{-}(x)=\left\{\begin{array}{cc}
-0.4 & x=0 \\
-0.3 & x=1 \\
-0.1 & x=2,3
\end{array}\right.\right.
$$

is a BVF-ideal of $X$ but not BVFC-ideal, since $\mu^{+}(0 * 1)<\mu^{+}(1)$ and $\nu^{-}(0 * 1)>$ $\nu^{-}(1)$.

Theorem 17. Intersection of any two BVF-ideals of $X$ is also a BVF-ideal.

Proof. Let $A=\left(X ; \mu_{A}^{+}, \nu_{A}^{-}\right)$and $B=\left(X ; \mu_{B}^{+}, \nu_{B}^{-}\right)$be two BVF-ideals of $X$.

Take $C=A \cap B=\left(X ; \mu_{C}^{+}, \nu_{C}^{-}\right)$

where $\mu_{C}^{+}(x)=\mu_{A}^{+}(x) \wedge \mu_{B}^{+}(x)$ and $\nu_{C}^{-}(x)=\nu_{A}^{-}(x) \vee \nu_{B}^{-}(x)$.

Let $x, y \in X$.

Now, $\mu_{C}^{+}(0)=\mu_{A}^{+}(0) \wedge \mu_{B}^{+}(0) \geq \mu_{A}^{+}(x) \wedge \mu_{B}^{+}(x)=\mu_{C}^{+}(x)$

and $\nu_{C}^{-}(0)=\nu_{A}^{-}(0) \vee \nu_{B}^{-}(0) \leq \nu_{A}^{-}(x) \vee \nu_{B}^{-}(x)=\nu_{C}^{-}(x)$

$$
\text { Also } \begin{aligned}
\mu_{C}^{+}(x) & =\mu_{A}^{+}(x) \wedge \mu_{B}^{+}(x) \\
& \geq\left(\mu_{A}^{+}(x * y) \wedge \mu_{A}^{+}(y)\right) \wedge\left(\mu_{B}^{+}(x * y) \wedge \mu_{B}^{+}(y)\right)
\end{aligned}
$$




$$
\begin{aligned}
& =\left(\mu_{A}^{+}(x * y) \wedge \mu_{B}^{+}(x * y)\right) \wedge\left(\mu_{A}^{+}(y) \wedge \mu_{B}^{+}(y)\right) \\
& =\mu_{C}^{+}(x * y) \wedge \mu_{C}^{+}(y) \\
\nu_{C}^{-}(x) & =\nu_{A}^{-}(x) \vee \nu_{B}^{-}(x) \\
\leq & \left(\nu_{A}^{-}(x * y) \vee \nu_{A}^{-}(y)\right) \vee\left(\nu_{B}^{-}(x * y) \vee \nu_{B}^{-}(y)\right) \\
& =\left(\nu_{A}^{-}(x * y) \vee \nu_{B}^{-}(x * y)\right) \vee\left(\nu_{A}^{-}(y) \vee \nu_{B}^{-}(y)\right) \\
& =\nu_{C}^{-}(x * y) \vee \nu_{C}^{-}(y)
\end{aligned}
$$

This completes the proof.

The above theorem can be generalized as follows.

Theorem 18. The intersection of a family of BVF-ideals of $X$ is a BVFideal of $X$.

One can prove the following analogously.

Theorem 19. Intersection of any two BVFC-ideals of $X$ is also a BVFCideal and hence, the intersection of a family of $B V F C$ - ideals of $X$ is also a $B V F C$-ideal of $X$.

Theorem 20. In a BF-algebra $X$, every bipolar valued fuzzy ideal of $X$ is a bipolar valued fuzzy subalgebra of $X$.

Proof. Let $B=\left(X ; \mu^{+}, \nu^{-}\right)$be a bipolar valued fuzzy ideal of a BF-algebra $X$. Since $x * y \leq x$ forall $x, y \in X$, it follows that

$$
\mu^{+}(x * y) \geq \mu^{+}(x) \text { and } \nu^{-}(x * y) \leq \nu^{-}(x) .
$$

so from (1) that $\mu^{+}(x * y) \geq \mu^{+}(x) \geq \mu^{+}(x * y) \wedge \mu^{+}(y) \geq \mu^{+}(x) \wedge \mu^{+}(y)$.

and $\left.\nu^{-}(x * y) \leq \nu^{-}(x) \leq \nu^{-}(x * y) \vee \nu^{-}(y)\right) \leq \nu^{-}(x) \vee \nu^{-}(y)$.

Hence, $B=\left(X ; \mu^{+}, \nu^{-}\right)$is a bipolar valued fuzzy subalgebra of $X$.

The converse of this theorem is not true in general.

Example 21. Consider the bipolar valued fuzzy subalgebra $B=\left(X ; \mu^{+}, \nu^{-}\right)$ in example 6. If $B$ is in BVF-ideal, then $\nu^{-}(x) \leq \nu^{-}(x * y) \vee \nu^{-}(y) \forall x, y \in X$ should hold.

Take $x=2$ and $y=4$

We have

$\nu^{-}(2)=-0.2$ and $\nu^{-}(2 * 4) \vee \nu^{-}(4)=\nu^{-}(3) \vee \nu^{-}(4)=-0.8 \vee-0.8=-0.8$.

So, $B$ is not BVF-ideal of $X$. 
Now, a sufficient condition is derived for a BVF-subalgebra to be a BVFideal as follows.

Theorem 22. Let $B=\left(X, \mu^{+}, \nu^{-}\right)$be a bipolar valued fuzzy subalgebra of a BF-algebra $X$ such that $\mu^{+}(x) \geq \mu^{+}(y) \wedge \mu^{+}(z)$ and $\nu^{-}(x) \leq \nu^{-}(y) \vee$ $\nu^{-}(z)$ holds for all $x, y, z \in X$ satisfying the inequality $x * y \leq z$. Then, $B=$ $\left(X ; \mu^{+}, \nu^{-}\right)$is a bipolar valued fuzzy ideal of $X$.

Proof. It is recalled from theorem 7 that $\mu^{+}(0) \geq \mu^{+}(x)$ and $\nu^{-}(0) \leq \nu^{-}(x)$ forall $x \in X$. Since $x *(x * y) \leq y$ for all $x, y \in X$, it follows from given condition that

$$
\mu^{+}(x) \geq \mu^{+}(x * y) \wedge \mu^{+}(y) \text { and } \nu^{-}(x) \leq \nu^{-}(x * y) \vee \nu^{-}(y)
$$

Hence, $B=\left(X ; \mu^{+} . \nu^{-}\right)$is a bipolar valued fuzzy ideal of $X$.

Remark 23. $B=\left(X ; \mu^{+}, \nu^{-}\right)$is a BVFS defined on any universe $X$, if and only if $\mu^{+}$and $-\nu^{-}$are the fuzzy subsets of $X$.

Theorem 24. A BVFS $B=\left(X ; \mu^{+}, \nu^{-}\right)$is a BVF-ideal of $X$, if and only if the fuzzy subsets $\mu^{+}$and $-\nu^{-}$are fuzzy-BF-ideals of $X$.

Proof. Let $B=\left(X ; \mu^{+}, \nu^{-}\right)$be an BVF-ideal of $X$.

Then, clearly $\mu^{+}$is a fuzzy-BF-ideal of $X$.

Also $\nu^{-}(0) \leq \nu^{-}(x)$ and $\nu^{-}(x) \leq \nu^{-}(x * y) \vee \nu^{-}(y)$.

$\Rightarrow-\nu^{-}(0) \geq-\nu^{-}(x)$ and $-\nu^{-}(x) \geq-\nu^{-}(x * y) \wedge-\nu^{-}(y)$.

Therefore, $-\nu^{-}$is a fuzzy-BF-ideal of $X$.

Conversely, Assume $\mu^{+}$and $-\nu^{-}$are fuzzy-BF-ideals of $X$. $X$.

It is enough to prove that $\nu^{-}(0) \leq \nu^{-}(x)$ and $\nu^{-}(x) \leq \nu^{-}(x * y) \vee \nu^{-}(y) \forall x, y \in$

$$
\begin{array}{r}
\text { For, }-\nu^{-}(0) \geq-\nu^{-}(x) \text { and }-\nu^{-}(x) \geq-\nu^{-}(x * y) \wedge-\nu^{-}(y) . \\
\Rightarrow \nu^{-}(0) \leq \nu^{-}(x) \text { and } \nu^{-}(x) \leq \nu^{-}(x * y) \vee \nu^{-}(y) .
\end{array}
$$

This completes the proof.

Using this theorem, the following are obtained.

Theorem 25. A BVFS $B=\left(X ; \mu^{+}, \nu^{-}\right)$is a BVF-ideal of $X$, if and only if the $B V F S \square B=\left(X ; \mu^{+},-\mu^{+}\right)$and $\diamond B=\left(X ;-\nu^{-}, \nu^{-}\right)$are also BVF-ideals of $X$.

Proof. $B=\left(X ; \mu^{+}, \nu^{-}\right)$be an BVF-ideal of $X$. 
if and only if the fuzzy subsets $\mu^{+}$and $-\nu^{-}$are fuzzy $B F-$ ideals of $X$, by above theorem,

if and only if $\square A$ and $\diamond A$ are also BVF-ideals of $X$ by the definition of $\square A$ and $\diamond A$.

Analogously, the following can be proved:

Theorem 26. An $B V F$ subset $A$ of $X$ is an $B V F C$ - $B F$-ideal of $X$ if and only if $\square B=\left(X ; \mu^{+},-\mu^{+}\right)$and $\diamond B=\left(X ;-\nu^{-}, \nu^{-}\right)$are also BVFC-BF-ideals of $X$.

\section{Conclusion}

In this work, the investigation of the Bipolar Valued fuzzy structures on BFalgebras is started and several interesting results are arrived. The surprising point is that Andrzej Walendziak[1] says that the structure of BF algebra becomes a BG-algebra, by the proof following directly from the definition. Hence, it is concluded that whatever result is proved for BF-algebras, can directly be carried over to BG-algebras.

\section{References}

[1] Andrzej Walendziak, On BF-algebras, Mathematica Slovaca, 57, No. 2 (2007), 119-128.

[2] Farhat Nisar, Bipolar-valued fuzzy K-subalgebras, World Applied Sciences Journal, 14, No. 12 (2011), 1914-1919.

[3] K.M. Lee, Bipolar valued-valued fuzzy sets and their operations, In: Proc. Int. Conf. on Intelligent Technologies ,Bangkok, Thailand (2000), 307-312.

[4] K.M. Lee, Comparison of interval-valued fuzzy sets, Intuitionistic fuzzy sets, and bipolar valued-valued fuzzy sets, Fuzzy Logic Intelligent Systems, 14, No. 2 (2004), 125-129.

[5] P. Muralikrishna, M. Chandramouleeswaran, Homomorphism on intuitionistic l-fuzzy BF/BG-subalgebras, Advances in Fuzzy Mathematics, 5, No. 3 (2010), 311316.

[6] P. Muralikrishna, M. Chandramouleeswaran, Study on N-ideals of BF-algebras, International Journal of Pure and Applied Mathematics, 83, No. 4 (2013), 607-612.

[7] L.A. Zadeh, Fuzzy sets, Inform Control., 8 (1965), 338-353. 